\title{
Variation characteristics of extreme temperature in Yunnan province and Guizhou province
}

\author{
Li Xirong ${ }^{1}$, Yang Dong ${ }^{1, *}, \quad$ Yi Wei ${ }^{1}$ \\ ${ }^{1}$ Northwest Normal University,Collegeof Geography and Environmental Science, Lanzhou, China
}

\begin{abstract}
Using the daily temperature data from meteorological stations in Yunnan province and Guizhou province and Guizhou province from 2000 to 2019, NCEP/NCAR re-analyzed the data on a daily basis, and analyzed the temporal and spatial characteristics of extreme temperature events in the study area. Research shows that the extreme temperature in Yunnan province and Guizhou province region is warming; the extreme temperature index is different in spatial tendency, and all have abrupt changes; the maximum monthly maximum temperature and the heat persistence index will continue to increase in the future, the number of cold night days and the cold persistence index are on a strong and continuous decreasing trend.
\end{abstract}

\section{Introduction}

In the context of global warming, the time interval between the occurrence of regional extreme temperature events is gradually shortening, and the scope of influence is gradually expanding, and the impact on ecology, biodiversity, and human life is further increasing. The IPCC Fifth Assessment Report pointed out that the global average surface temperature increased by $0.72^{\circ} \mathrm{C}(0.49$ $0.89^{\circ} \mathrm{C}$ ) from 1951 to 2012 , and $1980-2012$ was the warmest 30 years since the Industrial Revolution ${ }^{[1]}$. In terms of temperature, the average annual temperature growth rate in China in the 20th century reached $0.08^{\circ} \mathrm{C} / 10 \mathrm{a}$. This upward trend has become more obvious in the past 50 years. From 1961 to 2005, the temperature growth rate in China reached $0.28^{\circ} \mathrm{C} / 10 \mathrm{a}^{[2]}$. The temperature change in Southwest of China is not synchronized with global warming, and the sudden change of climate elements in high-altitude areas is earlier than that in low-altitude areas ${ }^{[3]}$. For nearly half a century, the annual average temperature in Southwest China has increased at a rate of $0.12^{\circ} \mathrm{C} / 10 \mathrm{a}$, which is lower than the national average temperature rise rate, but higher than the global average temperature rise rate ${ }^{[4]}$.

\section{Information and Methods}

\subsection{Overview of the study area}

The two provinces are located on the Yunnan-Guizhou Plateau in the southwest of China. The topography and geomorphology of the study area are complex and one of the most complex regions in China. Yunnan province and
Guizhou province is located in a corner of the southwest, bordering Sichuan Province and Chongqing City to the north, Hunan Province, the home of fish and rice to the east, Guangxi Autonomous Region, Vietnam, and Laos to the south, and Tibet and Myanmar to the west.The topography of the study area is plateau and mountainous. The topography of the territory is high in the northwest and low in the southeast. It descends step by step from north to south. The altitude is generally about $3000-4000 \mathrm{~m}$. The climate in the study area is a subtropical humid area with a subtropical monsoon climate. It is a plateau, and the climate varies significantly due to different altitudes and atmospheric circulation conditions.

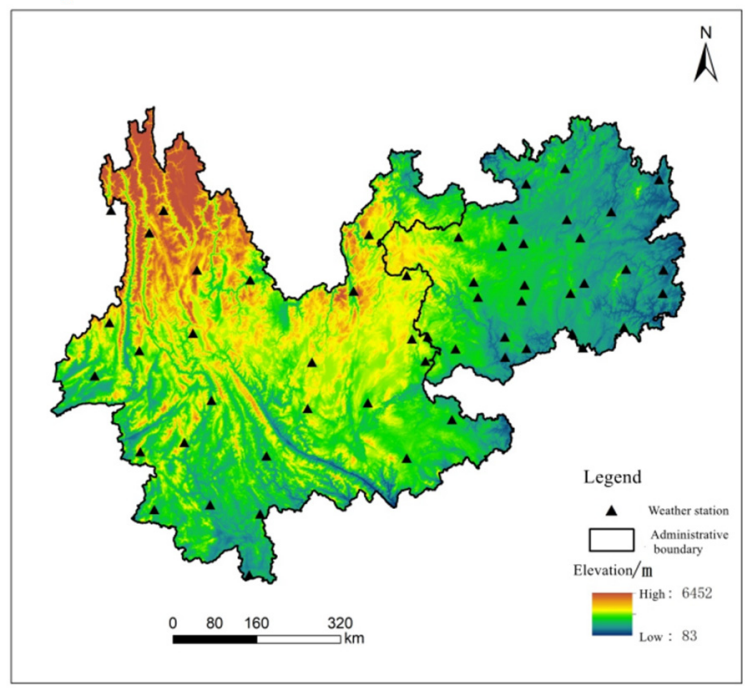

Figure 1 Overview of the study area

"Corresponding author: E-mail: yangdong@nwnu.Edu.cn 


\subsection{Data and research methods}

\subsection{1 meteorological data}

The meteorological data are derived from the National Meteorological Information Center of the China Meteorological Administration, which extracts the temperature data of 41 meteorological stations in the study area from 1960 to 2019.After sorting, get the required maximum temperature, minimum temperature, average temperature. Using the RClimDex model in R software to process the collated daily value data ,16 extreme temperature indexes were obtained from 2000-2019.

\subsubsection{Research methods}

\section{Linear Trend Method}

The linear trend method is a common method to study the change of extreme climate events. Time $(t)$ as independent variable and extreme temperature index $(y)$ as dependent variable. The relationship between time $(t)$ and extreme temperature index $(y)$ is established:

$$
y(t)=b_{0}+b_{1} t
$$

The regression coefficient $b_{1}$ reflects the rising or falling trend of extreme temperature indicators, with $b_{1}>0$ showing an upward trend, $b_{1}<0$ showing a downward trend. The absolute value of $b_{1}$ indicates the speed of upward or downward trend.

2.M-K mutation test

Mann-Kendall(M-K) test is a widely used nonparametric test method, which does not need samples to follow a certain distribution, nor is it disturbed by a small number of outliers. It is suitable for meteorological, hydrological and other non-normal distribution data, with strong applicability and convenient calculation. Time series $x$ contain $n$ sample size to build order columns:

$$
s_{k}=\sum_{i=1}^{k} r_{i}(k=2,3, \cdots, n)
$$

Assuming random time series independence:

$$
U F_{k}=\frac{\left[s_{k}-E\left(s_{k}\right)\right]}{\sqrt{\operatorname{Var}\left(s_{k}\right)}}(k=1,2,3 \cdots, n)
$$

Among them, $U F_{1}=0 ; E\left(s_{k}\right)$ and $\operatorname{Var}\left(s_{k}\right)$ are the mean and variance of the cumulative number $s_{k}$, and when $x_{1}, x_{2}, \ldots, x_{n}$ are independent of each other, they have the same continuous distribution, which can be calculated by the following formula:

$$
\begin{aligned}
E\left(s_{k}\right) & =\frac{k(k+1)}{4} \\
\operatorname{Var}\left(s_{k}\right) & =\frac{k(k-1)(2 k+5)}{72}
\end{aligned}
$$

After the time series $x$ is arranged in reverse order, the above operation is repeated to make $-U B_{k}=U F_{k}$. If $U F_{k}>0$, the series shows an upward trend, and $U F_{k}<0$ shows a downward trend. And given the significance level: when $\partial=0.05, U=1.96$ critical line. If there is an intersection between curves $U B_{k}, U F_{k}$ and it is between the critical lines, the abrupt start time is the intersection time ${ }^{[5]}$.
3.R/S analysis methodology

To study the statistical characteristics of time series, Hurst proposed a re-scalar difference analysis method (rescaled Range Analysis), in which the Hurst index can effectively judge the continuity of time series and predict its development trend ${ }^{[6]}$. The rationale is as follows:suppose a time series $x_{1}, x_{2}, \ldots, x_{n}$ (n represents the length of the time series), for any integer $s \geq 1$,

$$
\begin{gathered}
\text { Mean sequence: } \\
\bar{x}_{f}=\frac{1}{f} \sum_{i=1}^{f} x_{i}, \quad s=1,2, \ldots, n \\
\text { Cumulative deviations: }
\end{gathered}
$$

$$
\begin{gathered}
\mathrm{X}(i, f)=\sum_{m=1}^{f}\left(x_{m}-\bar{x}_{f}\right), \quad 1 \leq i \leq f \\
\text { Extreme: } \\
R_{s}=\max _{1 \leq i \leq f} \mathrm{X}(i, f)-\min _{1 \leq i \leq f} \mathrm{X}(i, f) \\
\text { Standard deviation: }
\end{gathered}
$$

$$
\begin{aligned}
& S_{f}=\left[\frac{1}{n} \sum_{m=1}^{f}\left(x_{i}-\bar{x}_{f}\right)^{2}\right]^{\frac{1}{2}}, f=1,2, \ldots, n \\
& \quad \text { Hurst index: } \\
& R_{f} / S_{f}=(c f)^{H}
\end{aligned}
$$

According to the value of $f$, the least square method is used to estimate $H$, when $H=0.5$, it means that the time series is completely independent and the future has nothing to do with the past; When $0<H<0.5$, it means that the future trend has anti-persistence with the past, and the smaller $\mathrm{H}$, the stronger the anti-persistence; $0.5<H<1$ indicates that the future changes of time series are consistent with the past, and the greater the $\mathrm{H}$, the stronger the persistence.

\section{Fruit analysis}

\subsection{Temporal and spatial variation of extreme temperature index}

The temporal trends of 16 extreme temperature indices in Yunnan province and Guizhou province region from 2000 to 2019 are shown in figure 2, The number of frost days (FD0), ice days (ID0), crop growth period (GSL), monthly maximum temperature minimum ( $\mathrm{TXn}$ ), cold night days (TN10p), cold day days (TX10p), cold duration index (CSDI) and monthly average daily difference (DTR) showed a decreasing trend, The rates of change were about $-0.58 \mathrm{~d} / 10 \mathrm{a} 、-0.02 \mathrm{~d} / 10 \mathrm{a} 、-1.85 \mathrm{~d} / 10 \mathrm{a} 、-0.33^{\circ} \mathrm{C} / 10 \mathrm{a} 、-$ $2.08 \mathrm{~d} / 10 \mathrm{a} 、-1.09 \mathrm{~d} / 10 \mathrm{a} 、-1.95 \mathrm{~d} / 10 \mathrm{a}$ and $-0.07{ }^{\circ} \mathrm{C} / 10$, respectively Among them ID0 reduction rate is weak, CSDI reduction is relatively high, And TN10p reduction trend passed 0.05 significance test. Of the eight extreme indicators showing a downward trend, Except for GSL, Other indexes belong to extreme temperature cold index. The number of summer days (SU25), the number of hot nights (TR20), the maximum monthly temperature (TXx), the minimum monthly temperature $(\mathrm{TNn})$, the maximum monthly minimum temperature (TNx), the number of warm nights (TN90p), the number of warm days (TX90p) 
and the heat duration index (WSDI) all showed an upward trend, The growth rate is about $6.94 \mathrm{~d} / 10 \mathrm{a} 、 7.96 \mathrm{~d} / 10 \mathrm{a}$ 、 $0.51{ }^{\circ} \mathrm{C} / 10$ a, $0.02 \mathrm{~d} / 10 \mathrm{a} 、 7.96 \mathrm{~d} / 10 \mathrm{a} 、 0.51{ }^{\circ} \mathrm{C} / 10 \mathrm{a} 、$ $2.76 \mathrm{~d} / 10 \mathrm{a} 、 2.15 \mathrm{~d} / 10 \mathrm{a} 、 2.07 \mathrm{~d} / 10 \mathrm{a}$, respectively SU25、 TR20 growth rates are higher than other extreme temperature indices, At the same time TR20、TNx、TXx 、
TX90p pass the 0.05 level test, These extreme temperature and heat index increases significantly, This is consistent with the upward trend in temperature since 2000 . TNx is an extreme temperature cold index, It shows a slight increase, About 23 days of annual TNx in Yunnan province and Guizhou province, Compared with the extreme temperature warming index, the increase is weak.
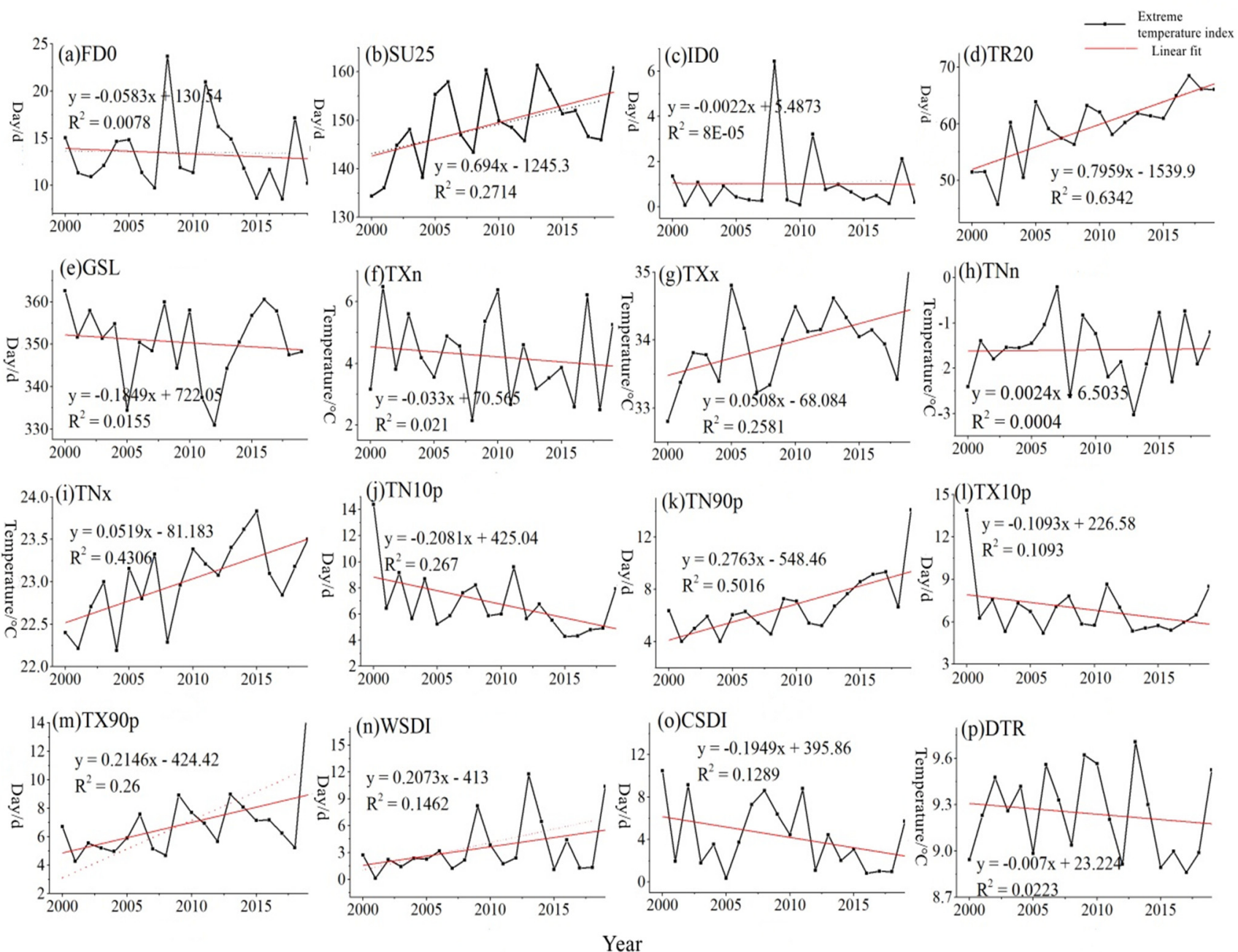

Figure 2 Trends in the time of extreme temperature index in Yunnan province and Guizhou province region ,2000-2019

Use ArcGIS Kriging interpolation and spatial mapping, the trend of spatial change is expressed. It can be seen from Figure 3 that there is a spatial difference in the extreme temperature index change trend in Yunnan province and Guizhou province area. The change trend of FD0 is $-2.5841-1.5078 \mathrm{~d} / 10 \mathrm{a}, \mathrm{FD} 0$ in the Yunnan province and Guizhou province, the overall trend of east-west increase in the central decline, SU25 in most parts of Yunnan, A maximum growth rate of about $15.4 \mathrm{~d} / 10 \mathrm{a}$, And SU25 in the western of the study area show a slight decline, It is increasing from east to west. TXx,TX90p,WSDI and DTR tend to decrease from west to east, These extreme temperature indices are concentrated in Yunnan, In Guizhou, Among them WSDI the trend of general increase in space, But the change is relatively high, An obvious WSDI increasing trend in the southwest of Yunnan province and Guizhou province; The increase of TXx,TX90p and DTR in central Yunnan is high.
ID0,TNn,TX10p increases in spatial variation from west to east, In the eastern part of study area, and in study area of Yunnan Province below the trend of decline. TR20 showed a marked increase in the southwest and northeast of Yunnan province and Guizhou province, $15.99 \mathrm{~d} / 10 \mathrm{a}$, maximum growth rate The increase trend in the middle and northwest of Yunnan province and Guizhou province is not obvious. GSL from southern Yunnan province and Guizhou province to northern, Higher spatial variability, And in most parts of Yunnan, In the northern part of Yunnan province and Guizhou province, About d/10a.10.41 TXn shows an increasing trend in Study area Northwest, In the south of Study area, the trend is mainly downward, Generally, the change from south to north increases; TNx,TN90p is increasing in space, The growth area is in the middle of Study area, Generally from the middle of the cloud to the east-west trend of decline; CSDI in space, The decreasing trend in the northwest and east is 
lower than that in the southwest of Yunnan province and (a)FD0

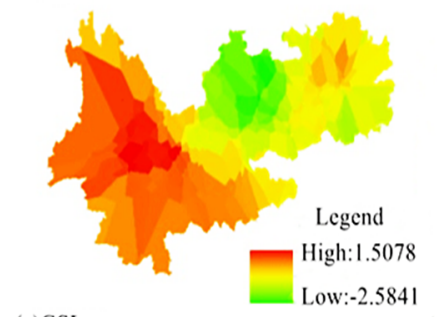

(c)GSL

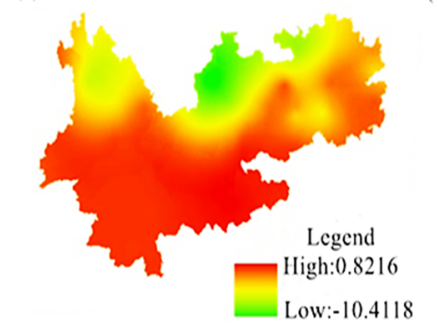

(i) $\mathrm{TNx}$

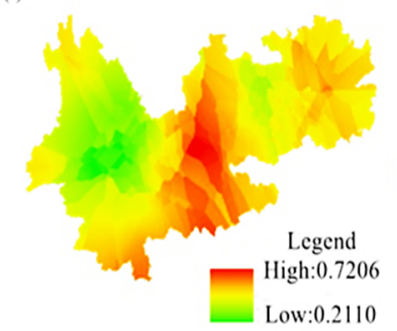

(m)TX90P

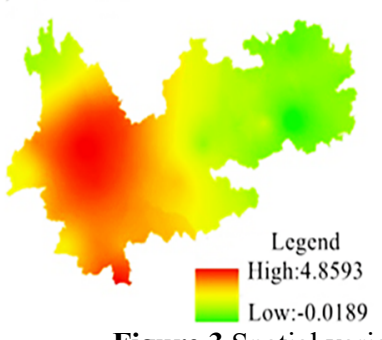
(b)SU25

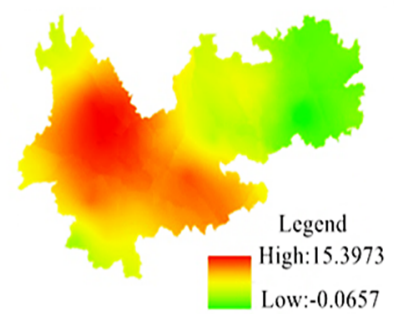

(f)TXn

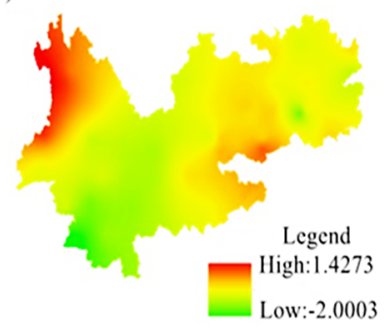

(j) TNIOP

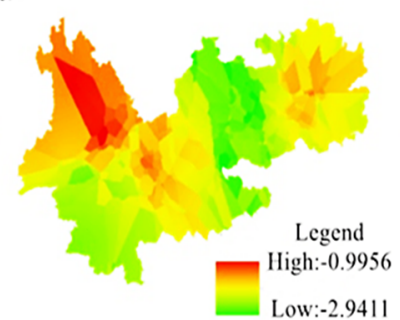

(n)WSDI

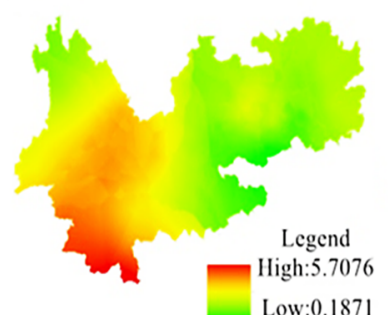

Guizhou province.

(c)ID0

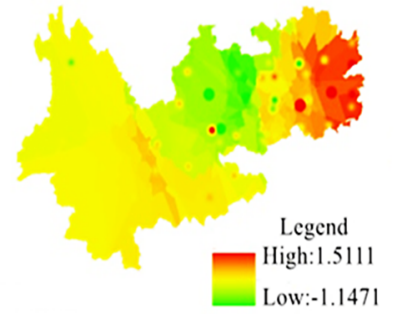

(g) $\mathrm{TXx}$

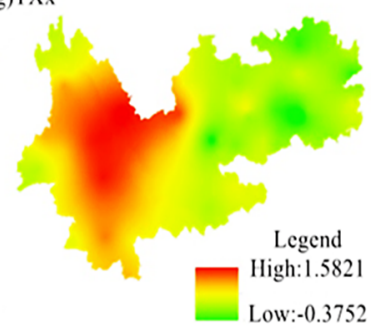

(k)TN90P

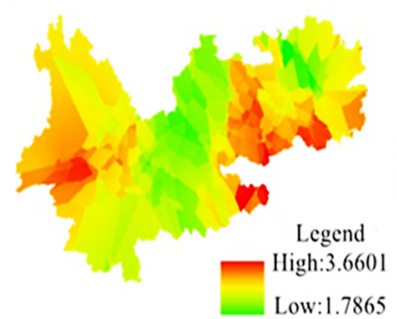

(o)CSDI

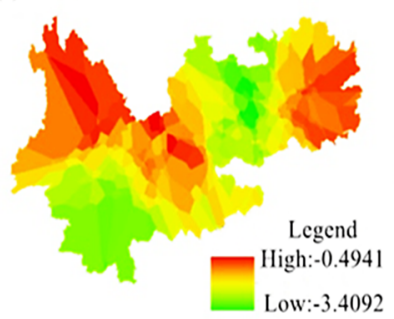

(d)TR20

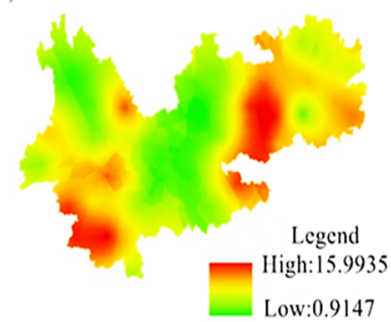

(h) $\mathrm{TNn}$

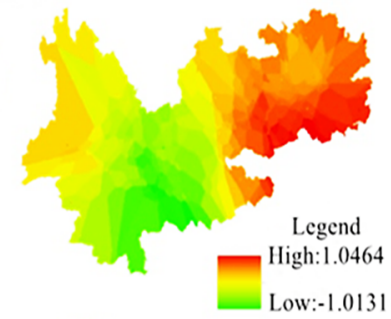

(1)TXI0P

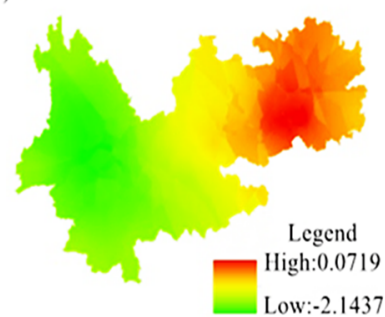

(p)DTR

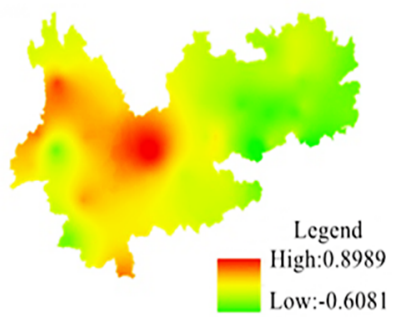

\subsection{Mutation analysis of extreme temperature indices}

Figure 4 shows the mutation test results of extreme temperature index in Yunnan province and Guizhou province area. Figure 3 shows that, there were mutation points in the extreme temperature index during the study period.FD0 has multiple mutations, and its fluctuations are obvious, especially after 2015; SU25 has a sudden change in 2002, and the UF curve has an obvious increasing trend after 2002. There are multiple mutation points in the ID0 study period, mainly since 2015 ; TR20 has a mutation in
2012, and TR20 has increased significantly since 2012. GSL had mutations in 2001, 2015 and 2017, and the trend of change was not obvious, while monthly TXn, TXx, TNx, TN10p, CSDI and TX90p had multiple mutation points before 2010, and TNn occurred multiple times after 2010 Suddenly, these indexes fluctuate significantly. The mutation year of TN90p was 2014, and there was a clear upward trend after 2014; TX10p only abrupted in 2001, and the trend of change was not significant; the mutation point of WSDI was concentrated after 2015, and the warm persistence index fluctuated strongly in recent years; There was a sudden change in 2014, and it has shown a downward trend since 2014. 

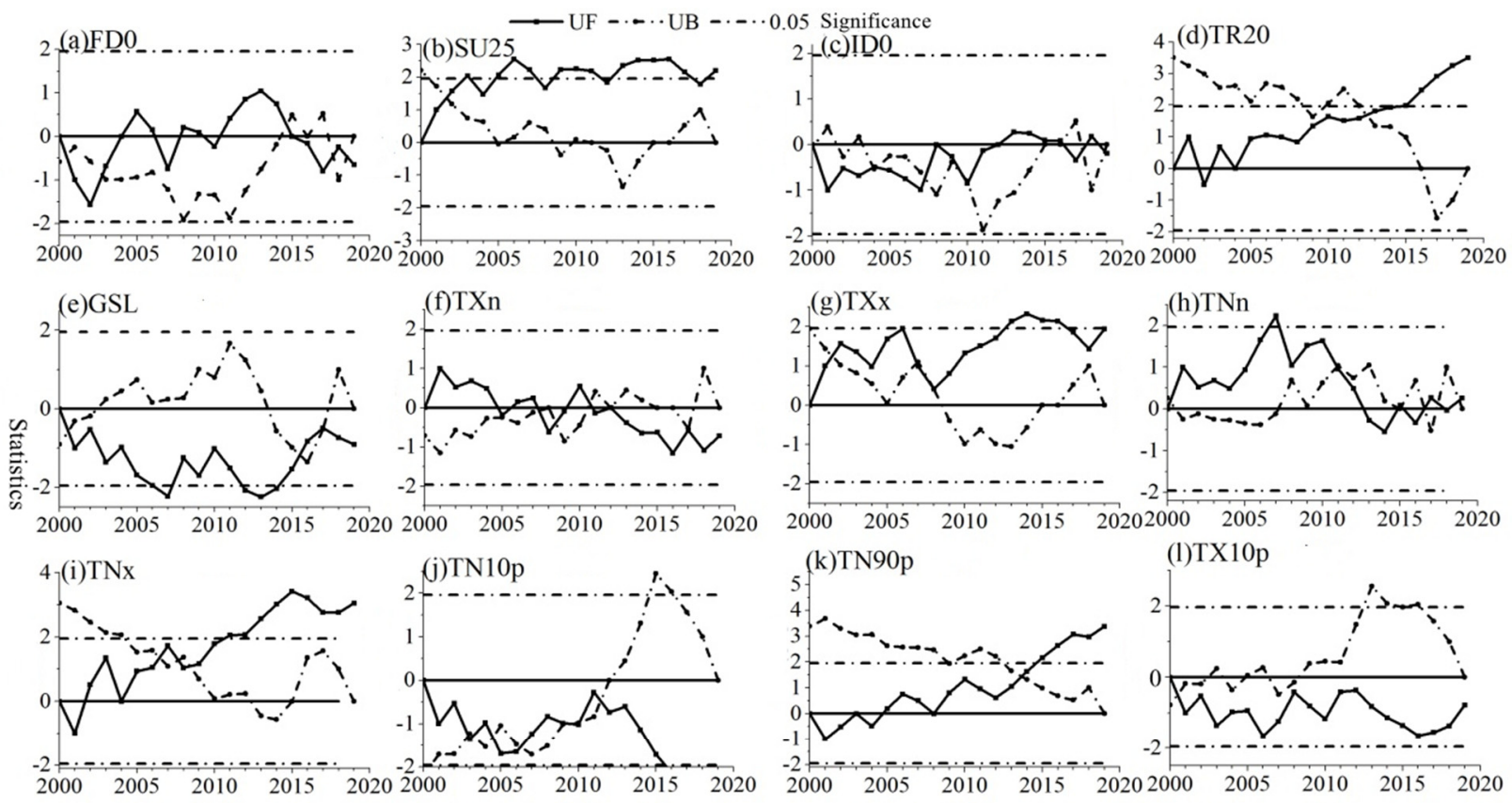

(1)TX10p
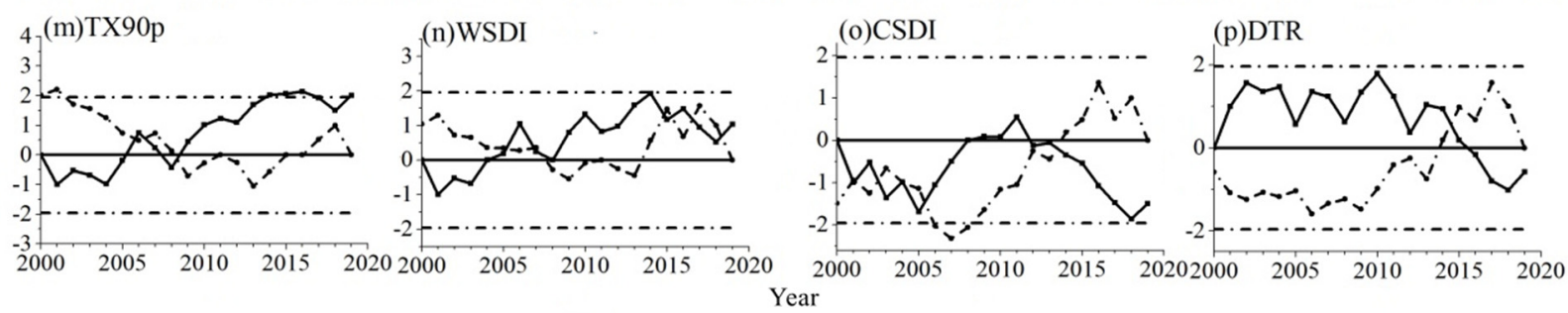

Figure 4 Mutation test of extreme temperature index in Yunnan province and Guizhou province area ,2000-2019

\subsection{Future projections of trends in extreme temperature indices}

With the aid of R/S analysis method, forecast the future trend of extreme temperature index in Yunnan and Guizhou area. It can be seen from Table 1 that there is a Hurst phenomenon in the extreme temperature index in Yunnan province and Guizhou province. The Hurst index of TXx, TN10p, TX10p, and WSDI is greater than 0.5 , indicating that the future trend is consistent with the historical trend, and the future TXx, TN10p, TX10p, and WSDI will increase and decrease respectively. , Decrease and increase trends. Among them, the Hurst index of TN10p is relatively high and has strong persistence. That is, the number of cold nights in Yunnan province and Guizhou province area will decrease in the future. In the index with the Hurst index less than 0.5, the future trend of the index and the historical trend are countersustainable. FD0,ID0, GSL, TXn, CSDI, and DTR will increase in the future, and DRT has strong anti-continuity, that is, the asymmetry of temperature difference between days will increase; SU25, TR20, TNn, TNx, TN90p, TX90p will show a decreasing trend in the future .

\section{Conclusion}

1. The number of hot night days, maximum monthly maximum temperature, maximum monthly minimum temperature, warm night days, and warm day days from
2000 to 2019 in Yunnan province and Guizhou province region increased significantly, while the number of cold days showed a significant downward trend, while extreme temperatures in Yunnan province and Guizhou province region showed changes In the warming trend, the growth rate of the extreme warmth index is generally higher than the decrease rate of the extreme cold index.

2. The extreme temperature index is different in the spatial tendency. The number of summer days, the maximum monthly maximum temperature, the number of warm days, the heat persistence index, and the monthly average daily difference show a decreasing trend from west to east. The number of days with ice and the monthly minimum temperature are decreasing. The minimum value and the number of cold days, on the contrary, the minimum value of the crop growth period and the monthly maximum temperature generally increase from south to north, while other indexes have insignificant trend changes.

3. The extreme temperature index has abrupt changes, the number of frost days, the number of frozen days, the minimum monthly maximum temperature, the maximum monthly maximum temperature, the maximum monthly minimum temperature, the number of cold night days, the cold persistence index, the number of warm days, and the heat The persistence index and the minimum monthly minimum temperature have multiple sudden changes, and the fluctuations are obvious; the number of summer days, hot night days, and warm night days show an increasing trend after the sudden change in 2002, 2012, and 2014, 
while the monthly average The daily difference (DTR) showed a downward trend after the 2014 mutation point.

4. In the future, the maximum monthly maximum temperature and heat persistence index will continue to increase. The number of cold night days and the cold persistence index will continue to decrease; the number of frost days, the number of frozen days, the crop growth period, the minimum monthly maximum temperature, and the cold The persistence index and the monthly average daily difference index are counter-sustainable and will increase in the future, while the number of summer days, hot night days, minimum monthly minimum temperature, maximum monthly minimum temperature, warm night days, warm days The number of days will also be antisustainable and will show a downward trend in the future.

\section{References}

1. IPCC. (2013) Climate change 2013:The physical science basis:The Summary for Policymakers of the Working Group I Contribution to the Fifth Assessment Report. Cambridge University Press, The UK.

2. Xu Y., Xu C H. (2012) Preliminary Assessment of Simulations of Climate Changes over China by China by China CMIP5 Multi Models. J. Atmospheric and Oceanic Science Letters 5(6) :489-494.

3. Ma Z,Peng J., Gao W.,et al. (2006) Climate change facts in Southwest China for nearly 40 years. J. Plateau Meteorology, 25(4): 633-642.

4. Qi D M., Zhou C Y., Li Y Q., et al. (2012) Analysis on the Causes of Climate Change in Southwest China. J. Plateau and Mountain Meteorology Research, 32(1): 35-42.

5. Xu J. (2014) Geometry. Beijing: higher Education Press, Beijing.

6. Yang J Y., Zhao C., Liu G S,, Xu Y. (2017) Hydrological sequence change trend analysis based on Mann-Kendall and R/S method-Taking Suzhou as an example. J. Water Resources and Hydropower Engineering, 48(02): 27-30 +137. 